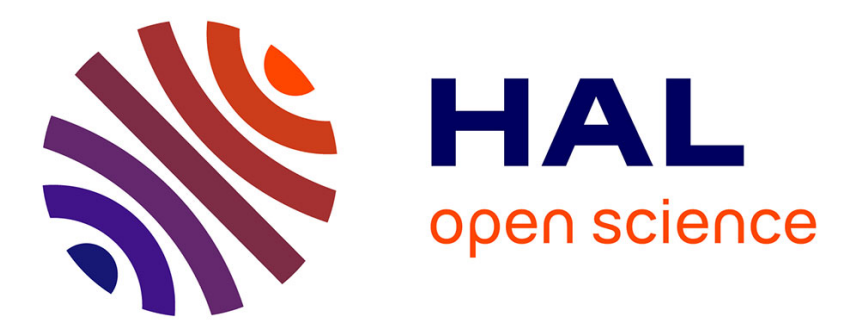

\title{
Un fragment inédit de bol signé [C. CINCI S]ENOVI[RI] découvert dans les ruines de l'aqueduc de Bavai, à Saint-Rémy-du-Nord
}

Jean-Louis Boucly

\section{- To cite this version:}

Jean-Louis Boucly. Un fragment inédit de bol signé [C. CINCI S]ENOVI[RI] découvert dans les ruines de l'aqueduc de Bavai, à Saint-Rémy-du-Nord. Gallia - Fouilles et monuments archéologiques en France métropolitaine, 1977, 35 (2), pp.263-269. 10.3406/galia.1977.1566 . hal-01939349

\section{HAL Id: hal-01939349 \\ https://hal.science/hal-01939349}

Submitted on 14 Jun 2020

HAL is a multi-disciplinary open access archive for the deposit and dissemination of scientific research documents, whether they are published or not. The documents may come from teaching and research institutions in France or abroad, or from public or private research centers.
L'archive ouverte pluridisciplinaire HAL, est destinée au dépôt et à la diffusion de documents scientifiques de niveau recherche, publiés ou non, émanant des établissements d'enseignement et de recherche français ou étrangers, des laboratoires publics ou privés.

\section{(ㅇ)(1) $\$$}

Distributed under a Creative Commons Attribution - NonCommercial - NoDerivatives| 4.0 


\title{
UN FRAGMENT INÉDIT DE BOL SIGNÉ [C. CINCI S]ENOVI[RI] DÉCOUVERT DANS LES RUINES DE L'AQUEDUC DE BAVAI, A SAINT-RÉMY-DU-NORD
}

\author{
par Jean-Louis BOUCLY
}

\begin{abstract}
Plusieurs éludes de .llle C. Bémont avaient permis de préciser que la période d'activité de SEVOIIR se siluail sous les regnes de Domitien et de Trajan². Or, un fragment de bol provenant d'orange fit supposer à .II. B. I)edet, J.-I. Fiches et E. Maistre 2 que ce décorateur avait pu travailler des l'époque de Vespasien en subissant probablement l'influence de Germanus. Contrairement a lous les autres framments de bols connus. qui sont décorés de métopes, relui d'(orange l'est en effet de zones paralliles ou ligurent. des motifs employés a la Ciraufesenque par Germanus.
\end{abstract}

Il restait à vérifier l'exactitude de ces supputations. Or, nous avons eu la chance de découvrir un lesson inscrit dont le style el cerlains motifs ressemblent beaucoup à ceux du bol d'Orangre. Verser au dossier ce document préciser quel en était le rontexte arrhoologique seront les deux objels de rette note.

1 6. BEmoxt, signalure inedile sur un moule de sigillie : (.. CINO SE.VOV ...), dans (iallia, XXVI, 1968, p. 301-313; lin potier ou décoraleur du sud de la Gaule : C.CINO SEVOOVIR ..., dans Gallia, XXVII, 1969, p. 186-205); Noles sur (C.CINO SF.VOFIRI, dans Gallia, XXIX, 1971, p. 200-217.

? B. DEDET, J.-L. Furhes el L. VAastre, Qualre bols Itr. 37 inédits du décorateur C.CIN , SENOVIRI dans Gallia, 33, 1975, p. 213-223.
En 1964, nous remimes au jour, à simintKémy-du-Nordª ce qu'il restail des soubassements d'un ou de plusieurs réservoirs disposés en amont du pont-aqueduc (fig. 1) qui franchissait la vallée de la simbre. Le ranal ou, plus probablement, les tuyaux de plomb que ce pont portait conduisait a Bavai les eaux raplées dans le bassin supérieur de La Tarsy ${ }^{5}$. Le dégagement des ruines puis l'exploration des sols antiques furent l'orcasion de nombreuses découvertes. $18 \mathrm{~m}$ de l'extrémité du piédroit $: 3^{6}$, par exemple, il y avait. jetés en tas contre le parement nord de la maçonnerie et liés par une sorte de mortier terreux, des tuileaux. de la limonite et des blocs de terre vitrifié auxquels se mêlaient des tessons de poteries. Parmi eux se trouvait le fragment de bol portant la signature incom-

3 R. Jolin ful l'insligaleur de ces recherches. les eludes havaisiennes lui doivent beaucoup et c'est un agreable devoir de lui temoigner iei toute notre rratitude. R. Jot.x, L'nqueduc de Floursies à Bavay. extrait des Annales du Cercle Archiologique de .Mons, I.XII, Gembloux, 19:5, p. xi3-x.4.

1 K. Jolin a dessine le plan. Nous lui devons aussi la restilution ideale des bassins.

5 Alluent de la rive droite de la Sambre. Certaines sources etaient captés a Floursies of a Dourlers arrondissement d'Avesnes-sur-Helpe.

6 l'3 l'indique sur le plan. 


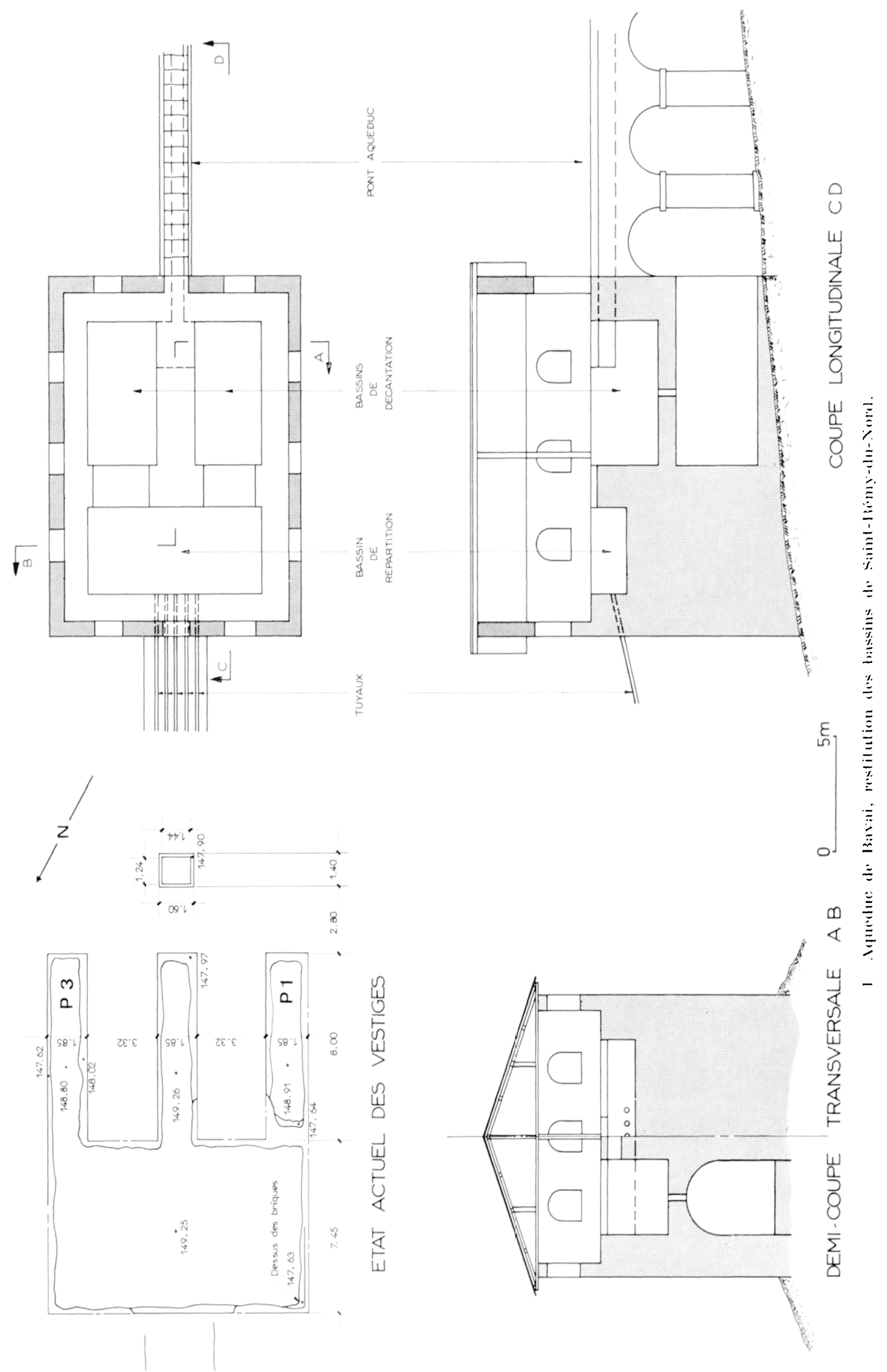



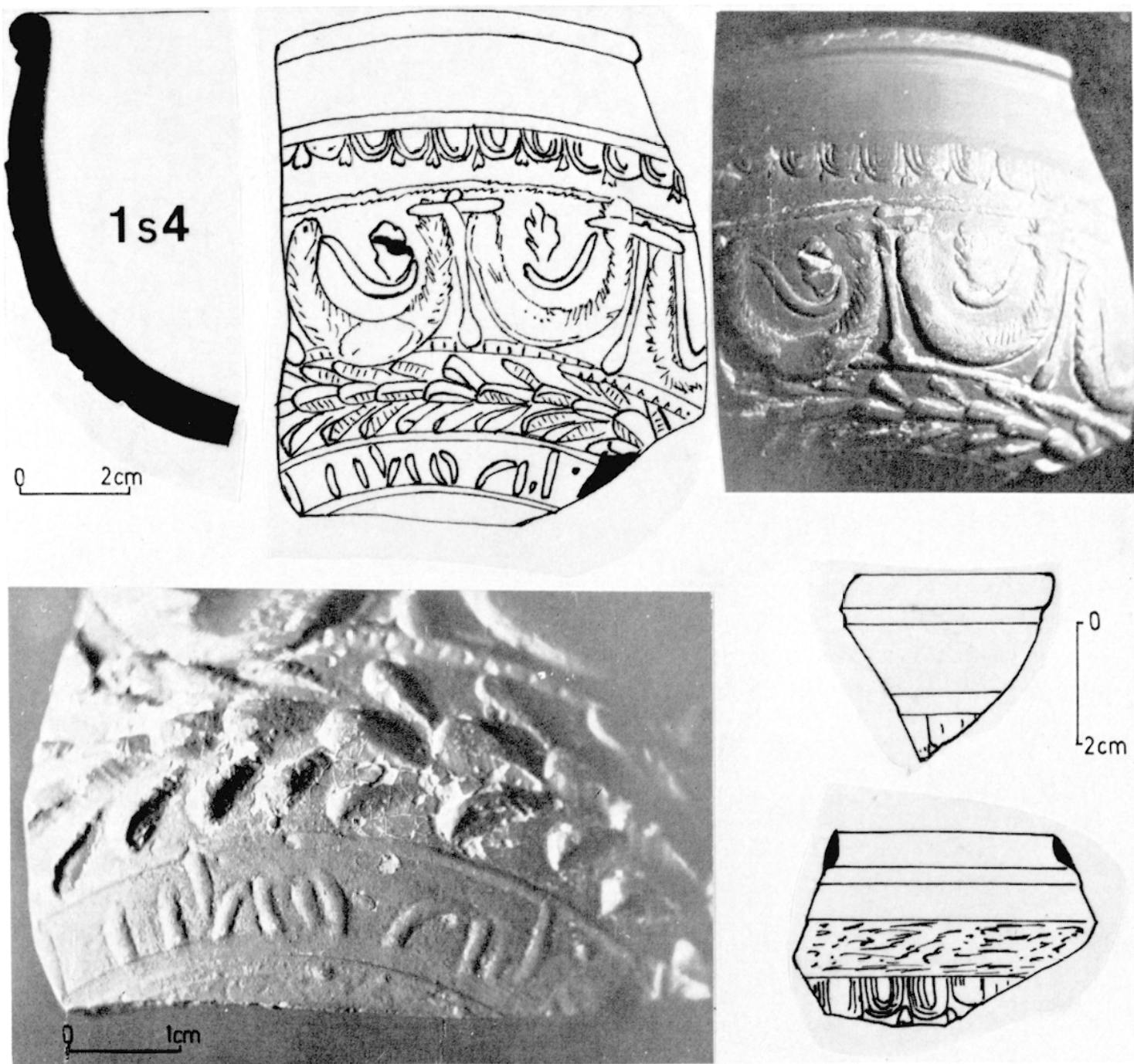

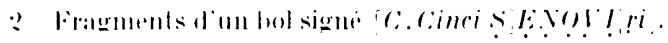

plete de SESOMIR tig. 2) Prolongere 7 m. plus a louest. la fouille liva ensuite deux auleres tessons plus pelits du meme vase. Ils se trouvaient sur le sol nalurel. arec des pierrailles, au niveau de lassise du premier des deux lis de moellons qu’il restail du parement 7 .

si le premier des lrois lesions domne toul le profil du vaissealu d'un bol hémisphérique de la forme 37 de Dragendortl. les deux autres ne présentenl quine partie du bord el de la frise d'oves. leur pale esl dure. homogrine. somore el fine, de rouleur rosece parsemere

7 Virs lat colla 116, ill. de nodules de chaux. Le vernis, de andeur marron oranger , est luisant. faiblement poreux. Il est generalement use sur les surfaces en fort relief el. par endroils, sobservent des écaillures el des reaguelures.

Ie vaissean n'a quane profondeur de $x \mathrm{~cm}$ environ. Le bord. de la ram de diamblee, est arrondi a sa partio superieure. Il saille vers l'exterieur oì il allecele la forme d'une petile baguetle ronvexe. I extremile inferieure de relle-ri est anguleuse. I n filed en relief ef un sillon, seblargisiant par endroits

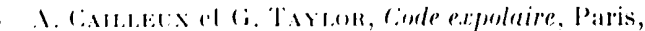
11". $13 x$. 
pour former une grorge, la bordent. Sous la partie unie du vaisseau, qui n'est pas toujours lisse, la frise d'oves est de forl mauvaise facture. sa largeur en effet n'est pas constante et les oves, imprimées de biais, ont perdu une partie de leur relief; elles sont petites. à cour étroit et à orles doubles. Le filet. i droite, n'est pas toujours visible et se lermine par une tète globulaire échancrée à gauche.

En dessous, s'étendent deux zones décorées dont la premiere est bordée par des cordons dentelés. Elle se compose de festons pennés, reliés asse\% gauchement par un jugum auquel s'attache un pendentif dont l'extrémité est ovale. I bans le champ que limitent le cordon supérieur el les festons, se trouvent une tige sinueuse tournée a droile puis a gatuche el une feuille de chène a cinq lobes. Mais dans le champ le plus a droite, qui est incomplet, on reconnait la tige plus longue et coudée qui figure sur le bol d'Orange.

la seconde \%one, limitée vers le bas par un filet en relief, est occupée par une guirlande trifoliée, sinistrogyre, dont certaines feuilles sont striées obliquement. Sous le décor. est imprimée a l'envers la signature rursive incomplete $S\left[E \mathrm{EOVI} r i_{\text {. }}\right.$. Elle ressemble a celle du bol is 49 de Salelles ${ }^{9}$, mais elle est plus large et plus régulière. C'est qu'elle s'integre ici dans la composition équilibrée du décor. Nous remarquons, en effet, que la surface extérieure du bol se divise en trois \%ones horizontales de même largeur. Ia première comprend la partie unie du vaisseau et la frise d'oves, de $14 \mathrm{~mm}$ chacune. si la seconde est couverte par les festons, la troisieme l'est par la gruirlande et la signature dont les largeurs respectives sont de 18 et de $10 \mathrm{~mm}$. Il en résulte une impression d'équilibre a laquelle concourt le rythme régulier des frises. le jeu de leurs lignes contrastées, leur largeur plus ou moins arande selon la position qu'elles occupent sur les flancs galbés du vaisseau créent un mourement harmonieux que gène malheureusement l'empatement des motifs. Ainsi done, le décor du tesson de saint-Kémydu-Nord, comme relui du bol d'orange.

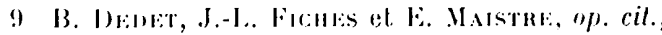
p. 214
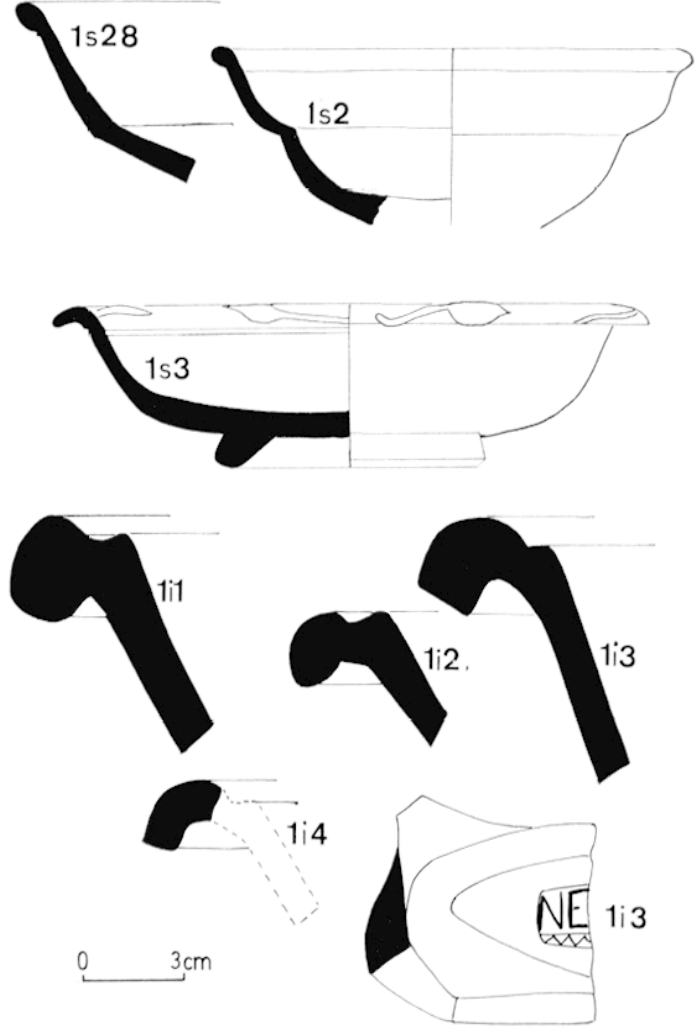

3 Profils : assiettes, bol et mortiers.

obrit a des principes de composition el porte la marque diun style différent de reux que l'on avait acroutumé d'altribuer a SESOTIR. Faut-il en ronclure avec M.I. B. Jedet. J.-I. Fiches el li. Maistre que de telles auvres, oil se manifestent des inlluences du début de l'époque flavienne. sont effectivement plus anciennes que les autres? Pour répondre i rette question. nous allons préciser maintenant le contexle de notre découverte.

Jans le dépot qui contenait le frament de sigillée signé, furent trouvés les tessons de vases suivants:

L ssielle de la forme 18 on $18 / 31$ de I)ragendorff (fig. :3). Quatre framments recollés (I S 28) appartiennent a la partie haute du vaisseau et du bord. La paite el le vernis ont les mèmes raractéristiques que relles du bol précédent. le bord, arrondi, a, vers l'extérieur, la forme d'une baguette convexe, de $24 \mathrm{~cm}$ de diametre,

10 Op. cil., p. 22:3. 
dont la base est creusée par un sillon. Celle forme de transition est fréquente durant la période I)omitien-Mardrien"1".

Assielle de la forme 36 de I ragendorff (fig. 3). Trois fragments recolles (I \& :3) donnent le profil presque complel de l'assielle. le vernis. plus orangé et luisant, parait indiquer une origine lédosienne. Il est très usé sur la surface intérieure du fond. I a paroi ivasce se relive en s'incurvant et se termine par un rourt marli convexe, coudé vers l'exlérieur. décoré de feuilles cordiformes, dont le diamitre est de 18 cm. In sillon le souligne vers l’intérieur. Le pied évide a une forme annulaire. (In peut rapprocher le profil de celle assielle de relui des nos 7, 12 (pl. I.III) d'()swald, dalés de la période Inomitien-Hadrien.

Bol de la forme 20 de Iraypendorff fig. :3). six fragments recollis (I 5 ? permeltent de reconstituer la majeure partie d'un bol bilobé. I.e fond et le pied manquent. le vernis. luisant, a une couleur rouge orange ; la pàte. dure, rosée, est sonore. Le bord, souligne vers l'intérieur par un sillon el arrondi i sil partie supérieure, forme vers l'extérieur une étroite baguelte convexe de $14 \mathrm{~cm}$ de diamil re. A rapprocher d'Oswald ${ }^{12}$, provenant de Pfün\% et datant de la période Trajan-Madrien.

Morliers en céramique jaume (fig. :3). Deux lypes de mortiers peuvent âlre distingues d'apris le profil de la colleretle qui se developpe vers l'extérieur du bord. Elle peul avoir la forme d'un boudin de section ovalaire, bien dégage de la paroi (1 i 1,1 i 2 , ou alre courle. en quart de cercle, aver une extrémilé plate surplombant le vaisseau ( 1 i :3, 1 i 4). Les plus grands spécimens ont une ouverlure de $34 \mathrm{~cm}$, les plus pelits ( 1 i 2,1 i 4), de 24 . I e premier type pourrait être dérivé de Colchester 19:3 Aa. daté de Néron. Il s'apparente al $n^{\circ} \quad 29$ (pl. IXXXVII) de K. Hartley, provenant du Kent, en usage pendant les années $9(1-1: 30$ ap. J.-C. Il existe encore vers la fin du ne siecle

11 J. (Oswaten et T. D). PRYck, In Introduction ln the study of Terra sigillala, Lomdres. 1966, pl. XI. I, $\mathrm{n}^{\circ} 3$.

1:3 Op. cil., pl. XI.IX, $n^{\circ} 19$. comme lattestent les nos :316 et :317 d'Arentsbur.oris

Le deuxime type marque probablement l’ivolution de la forme 22 de Wroxeter ou $3: 2$ de Richborough appartenant a l'époque Ciron-Vespasien ${ }^{14}$. Il s'apparente au $n^{0} 56$ de K. Hartley ${ }^{15}$, daté des années $70-110$ ap. J.-C. et annonce la forme lise de Gose, du premier liers du I $^{\mathrm{e}}$ siecle $^{16}$. Confirmerait celte datation le fait que l'un des 21 fragments numérotés 1 i :; présente, près du déversoir. le début d'un rartouche rectangulaire. bordé vers le has par un décor en dents de scie. oi figurent les lettres . VE/. Il sagit, en effet, du débul de la marque du polier NERICals dont la période d'antivité peul se situer durant le dernier tiers du I er siecle el le premicr quart du II $^{\mathrm{et}}$.

En définilive, celle élude nous permel de fixer la date du dépot dans les limites d'une périorle allant de Domitien a Madrien. si l'on admeltail toutefois que le fragment de bol décoré par SEYOI IR est contemporain des aulres tessons examines, il faudrait sans doute réduire cette période aux règnes de Domitien et de Trajan. Il nous semble au contraire que te morceau de bol signe est plus ancien que les autres tessons de la mème couche. Celui-la, en eflet. a pu se trouver an mème niveau que les deux aulres fragments du bol découverts

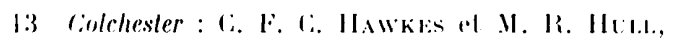

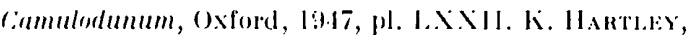
The Vorlaria and their origins, Fiflh report on the recalations of the Roman fort al Richborough, lient, dans Reports of the Research Commiltee of the Sorc of antiquaries of london, XXII, oxpord, 1968, p. 172-18:3.

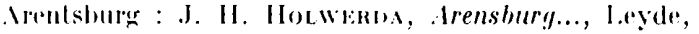
1923, pl. I.XI1.

1. Hroreler : Besur-lox, Excal'ations on the sile of the Roman lom'n at llroreler, shropshire, in 1912, dans Reporl of the Reseurch rimmillee of the soc. of 1nt. of London, 1, ()xford, 1913, p. 76, fig. 19. Richborou!gh : Bt:sll:-lox, Third report on the excavalions of the Roman fort al Richborough, Kenl, dans Reports of the Research commillee of the sor. of Anliquaries of london X, 1932, pl. XI.I.

15) Ol. cil.

l6 V. Cosss, liefässtypen der rämischen lieramik im Rheinland, Bomn, 1950), pl. XI.111.

17 II. Hexald of P. Innchl, Les marques de poliers a Bata!y, dans Pro Nervia, 11, 2, Ivesnes, 192:), p. $115-119$. 

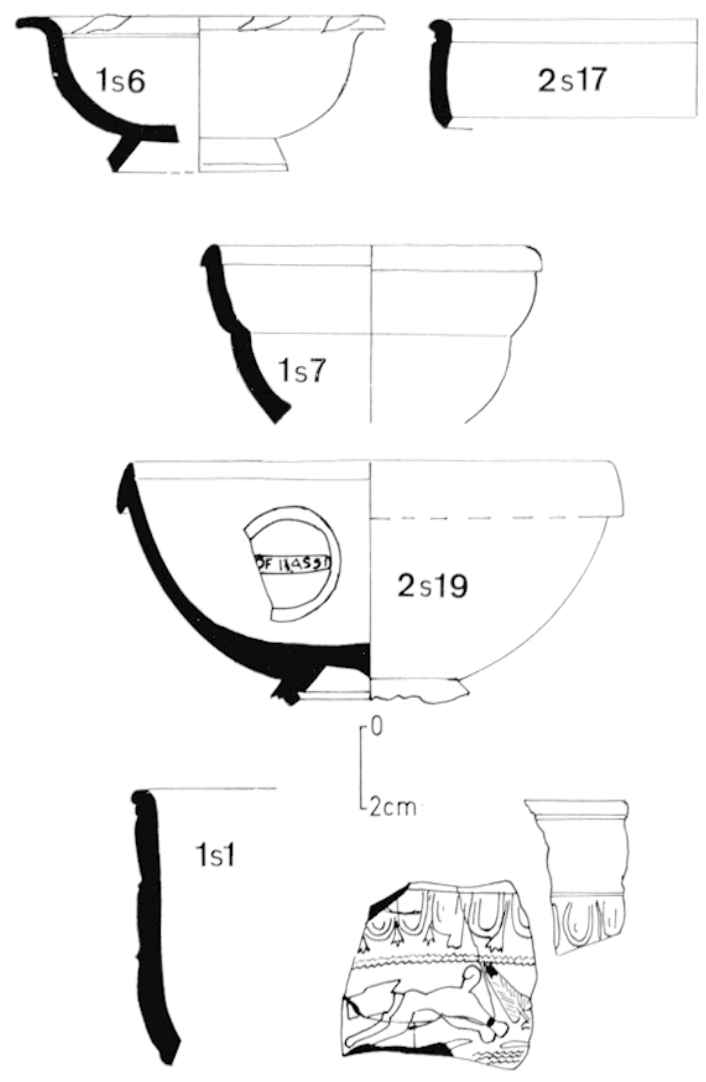

1 Profils : lasses al bol.

plus a l'ouest, puis alre ramasse el jeté parmi les debris accumules contre le parement de P:?. s'il en était anosi, on pourail penser que les trois morceatux du bol signe sonl ronlemporains de la ronstruction des hassins ed non pas posterieurs a elle. Or. lexploralion straligraphique des sols a permis de préciser d'une maniere assez sure le lerminus a quo de celle construction. Nous arons, en eftel, mis au jour, a un niveau plus bas que relui des restes apparents de maconneries. une autre couche archeologique et d'autres vestiges, témoins d'un premier etat que datent en particulier les tessons sllivants:

Tasses de la forme 2ơ de Mragendorff (fig. 1). lin frament ! 1 s 7) domme le profil jusquatu pred d'une tasse bilobere. la pale est dure. sonore. le vernis. luisint. a une couleur

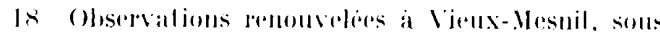
les vestiges doun bassin silur an ambnl diun aulme pont-afuedur. marron rouge ${ }^{19}$. Le bord, droil, arrondi à sat partie superieure, a vers l'extérieur une section prespue triamgulaire. Son diametre est de 10 rm. La forme parliculiere du bord. la presence d'un sillon vers l'intérienr, l'étroitesse du vaissean paraissent indiquer une époque asse\% haule, pourant s'étendre de Claude it Vespasien. Deux petits fragmenls $(2$ s 17$)$ donnent, le profil du lobe superieur d'une tasse plus grande. I e vernis est plus fonce el luisant. I.e hord. peu developpe, a un protil aigu vers l'extérieur oi il est limité par un filet en relief. son diamelre est de 14 cm.

Tasse de la forme 3ij de lmagendorff (fig. 4). six fragments ( I s (i) permeltent de reconstituer la majeure partie d'une tasse dont la pale. de rouleur rosée, est fine el rontient de petites lamelles de mica hanc. le vernis rouge orangé, est luisint. Ia paroi bien galbee se lemine par un court marli comvexe, coude vers l'exterieur el décoré de feuilles cordiformes. Vers l’intérieur, un sillon en marque la hase. I.e fond manque ; le pied conique evidé a son extrimite biseautee. Le diametre du bord est de 10 rm. II s'agil probablement d'un spereimen ancien de la forme 35) qui peut are dale de la pérode Vespasien-lomilien et rapproche d'(lswalde.

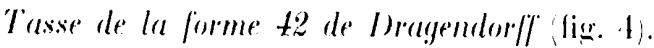
Huit fragments 2 s 19 ) permetlent de reconstiluer en partir une lasse dont la forme, rare dans nol re récion, apparait a lépoque flavienne a Joflueim Ritterling 1.12) ${ }^{21}$. Le vernis, luisant. " une rouleur marron rouge,22. Le bord est droit, arrondi a sa partie supérieure. souligne vers lintérieur par un sillon. Il aflecte vers lextérieur la forme dou bandeau légerement ronvexe. Des oreilles de prehension s'y allachaient comme le montre l'extrémilé conservé de l'une d'elles. Les flances sont assez galbes al le fomd, un peu ombiligue vers l'extérims.

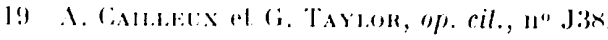

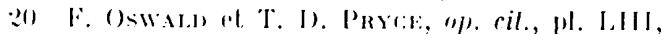
$11, \because-3$.

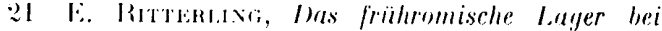
IInfheim im Tannus, dans Annalen des lereins für

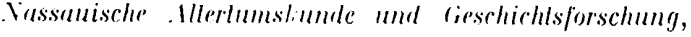
N1.. 1913.

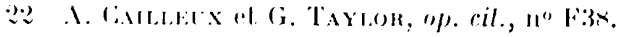


posait sur un pied en parlie brise. creuse par une large gorge. Vers l'intérieur, il est concave et porte, dans un cartouche rectangulaire entouré par un sillon rirrulaire. la marque (OFI ASSI. I n petil defaul d'impression a rérluil la lroisieme leltre a une haste verticale, re qui exclut la leclure of c:LSSSI et aulorise au contraire celle of B.LSSI ou

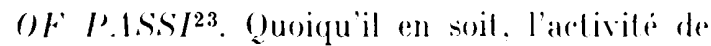
ces deux potiers de la Graufesengue na a pas dépassé le rigne de Vespasien.

Bol hémisphérique de la forme 37 de IOragendorff (tix. 4). Deux fragments (1 s l) proviennent de la partie supérieure du vaisseau et du bord d'un bol hemispherique déoré. Ie bord, tres peu développé vers l'exterieur. ef de 18 an de diamitre est limile par une gorore et par un filel en relief. Ia partie unie du vaisseau est légèrement convexe. Ia frise d'oves, assez large. est bordée vers le haut par un filet en relief el, vers le bas. par un cordon dentelé. les oves ont un cour elroit el allongé. des orles doubles; le filet, a droite, se lermine par une tèle trifide. Leur relief est assez flou comme celui de l'ove 32 de Hermet ${ }^{24}$ a a laquelle elles ressemblent beaucoup.

Ce qu'il reste du décor permet de reconnaitre une chasse llabellée. limilée vers le bas par un double cordon dentelé. In chien, tète assez carrée, queue relevee courte el louflue, court a gauche. I sa droite, une palmette oblique. comme relle des flabella $n^{\text {os }} 1$, (pl. (is) de llermel. s'y ajoule une végetation stylisée par de courles lignes ondulées, comme

$2: 3$ li. (J)wald), Inder of l'oller's' slamps on Terrt Sigillala, Margidumum, 19:31.

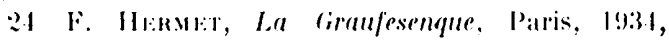
1). 35 his. au no 1:; (pl. (i7) du mème auteur. Par ses éléments et son style, le décor de ce bol peut itre dale de la période de transition de la Graufesenque. Précisons que le rordon dentelé, la tète trifide. la palmeltes se retrourent identiques sur un tesson de Xeusis daté des annees 7(1)-8i). sur lequel figure aussi un rhien courant asse\% semblable au notre.

Linsi donc, l'examen de ces donnes permet de fixer au début du réne de Vespasien la date des premieres constructions de l'aquedur de Barai. el à relui de Domitien le lerminus a quo des margonneries du second etal. Celles-ci. romme les fragments du bol de sisiollo trouves au niveau supérieur de leurs fondalions, seraient ainsi de l'époque DomitienTrajan.

Ces indications chronologiques corroborent parfailement les conclusions de llle C. Bémont sur la période d'activité probable de SE.TOI IR II faudra cependant d'autres découvertes pour apporter le romplément dinformation indispensable el faire que ces indices deviennent ou non une preuve. Quoiqu'il en soit, les trouvailles de saint-Rémy-du-Nord allestent que les relations commerciales entre la cité des Verviens el les potiers de terre sigillée du sud de la (iaule furent aussi llorissantes durant le dernier quart du ier siecle qu'elles l'avaient élé sous les empereurs Julio-Claudiens. P. Iarche, M. Hénault et le chanoine Hermet $y$ avaienl deja particulièrement insistere.

Jean-Iouis Bolicts.

25) (ion T. Wany, Jie sü̈lyallische Terra sigiliata ans . Velss, dans . Voraesium I, Limesforschmngen Band 6. Herlin, 1967, pl. :26, "10:1 r. p. 150.

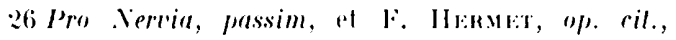
1. $2366-2: 37$. 